giemangel und Müdigkeit [...] mit einer messbaren Energiestoffwechselstörung in der Zelle.« (Ebd.)

Außer den genetischen oder biologischen stressbedingten Faktoren, die das Burnout-Syndrom auslösen und fördern können, existieren auch zahlreiche exogenen Faktoren, die vor allem im Bereich der Arbeit zu identifizieren sind.

\title{
2 Arbeitssoziologische Beobachtungen zum Burnout-Syndrom
}

Eine wesentliche Rolle bei der Erhöhung des Stressniveaus schreiben die Arbeitssoziologen zum einen den gesamtgesellschaftlichen Entwicklungen der letzten Jahre zu. Hier gehören Tendenzen wie der »Primat der Ökonomie, anhaltende hohe Arbeitslosigkeit, Arbeitsplatzunsicherheit, diskontinuierliche Erwerbskarrieren.« (Weber \& Hörmann 2007: 22) Zum anderen erhöhen das Stressniveaus prekäre und unsichere Lebensumstände, wie »schwere körperliche Erkrankungen, pflegebedürftige Angehörige, Scheitern/Instabilität von Beziehungen.« (Ebd.) Auch arbeitsbedingte psychosoziale Belastungen, wie etwa »chronischer Stress bei defizitärer sozialer Unterstützung, Arbeitsverdichtung, Betriebsklima, Konflikte, fehlende soziale Kompetenz von Führungskräften, ständige Erreichbarkeit, gestörte Work-Life-Balance« (ebd.) tragen zur Erhöhung des Stresses bei. Damit wird die enge Verbindung "zwischen negativem chronischem beruflichem Stress und dem Auftreten psychischer Störungen, insbesondere depressiver Erkrankungen« (ebd.) und ihre Auswirkung auf die steigende Arbeitsunfähigkeit und Frühinvalidisierung auffällig (vgl. a.a.O.: 24).

\subsection{Fehlpassung und Übermaß an Freiheit}

Aus arbeitssoziologischer Perspektive liegen die Ursachen oft auch auf der individuellen Ebene, und zwar in der "Nichtpassung zwischen den Eigenheiten der Person und den Gegebenheiten der Arbeitsaufgabe bzw. denen der Arbeitsbedingungen." (Hofmann 2015: 3f) Demnach ist nicht nur die Arbeitsbelastung ein relevanter Grund für die Entwicklung einer psychischen Störung, sondern auch »der Grad der Selbstbestimmung, der erlebten Sinnhaftigkeit der Arbeit und die Arbeitsbedingungen.«(Ebd.) Hierin wird die (An-)Passung der Tätigkeitsstruktur und der persönlichen Orientierung des Individuums hinterfragt. Die Beobachtung dessen, wie sich die Person der konkreten (Berufs-)Situation anpassen oder nicht anpassen kann, wird durch das sog. Peter-Prinzip beschrieben ${ }^{7}$, welches sich auch auf die 
Entstehung des Burnout-Syndroms übertragen lässt. Das Burnout-Syndrom tritt demnach dann auf, wenn

»die Kombination der RIASEC-Faktoren ${ }^{8}$ der Arbeitsaufgabe nicht der Kombination der RIASEC-Faktoren der Person entspricht. Das heißt, alte Erfolgsmuster führen in der neuen Tätigkeit auf der anderen Ebene nicht mehr zu Erfolgen, die zentralen Ängste der Person werden durch die Eigenarten der Tätigkeit aktiviert, die zentralen Bedürfnisse der Person können durch die Eigenarten der Tätigkeit nicht befriedigt werden, die Vorstellungen des Teams passen nicht zu der eigenen Person, die Kultur der Organisation weicht stark von den eigenen Vorstellungen zur optimalen Kultur ab oder die Konstellation Mitarbeiter/Vorgesetzter führt absehbar zu Konflikten.«(Ebd.)

Das Individuum muss daher selbst die Stufe der Fehlpassung erkennen, auf der die ausgeübte Tätigkeit Stress erzeugt und zum Burnout-Syndrom führen kann (vgl. a.a.O.: 215).

Neben der Fehlpassung des Individuums an die Arbeitsstruktur gibt es auch einen gegensätzlichen Fall - zu viel an Arbeitsfreiheit (vgl. Glißmann \& Peters 2001). Laut dem Stressreport der Bundesanstalt für Arbeitsschutz und Arbeitsmedizin geben zwei Drittel der Beschäftigten an, »bei der Arbeitsplanung und -einteilung über Handlungsspielraum zu verfügen, und über die Hälfte können selbst entscheiden, wann sie Pause machen.«(Ducki 2016: 83) Die existierenden Freiheiten wirken sich jedoch nur dort positiv aus, »wo Zeit- und Materialpuffer bereitstehen« (ebd.), was je nach dem Betrieb variieren kann. Daraus können die Arbeitssoziologen folgende Feststellung machen: »Mitarbeiter in modernen Unternehmen hätten heute nicht mehr das Problem fehlender Freiheiten, sondern litten sogar unter einem Zuviel davon. Die Qual der Wahl mache den Beschäftigten mehr Druck als klare Vorgaben durch das Management.« (Ebd.) Wenn im Ergebnis der Handlungsspielraum mit Beliebigkeit verwechselt wird und wenn die etwas vage formulierten Ziele durch die Mitarbeiter selbst erreicht werden müssen, dann »entsteht kein Spielraum, sondern Überforderung.« (Ebd.) Wird dieser Überforderung nicht entgegengewirkt,

ner Ebene/in seinem Bereich gut ist. Man wird ihm daher Potenzial für die Übernahme von Aufgaben auf der nächsten Ebene/in einem anderen Bereich zusprechen. Sofern er auch auf dieser nächsten Ebene/in diesem anderen Bereich gut ist, wird er für die Übernahme einer Funktion auf einer weiteren Ebene/in weiteren Bereichen vorgeschlagen usw. Irgendwann wird jedoch der Fall eintreten, dass er nicht mehr über die notwendigen Fähigkeiten auf der jeweiligen Ebene/in dem jeweiligen Bereich verfügt. Er hat dann, wie Peter es formuliert, die stufe seiner Inkompetenz، erreicht. « (Hofmann 2015: 214) forschend, A - artistic, künstlerisch, gestaltend, kreativ, S- social, helfend, erziehend, pflegend, E- enterprizing, führend, verkaufend, unternehmerisch, C- conventional, ordnend, verwaltend (vgl. Hofmann 2015: 54f). 
erzeugt sie Stress und kann das Ausbrennen verursachen. Mit anderen Worten, wurde früher die mangelnde Gestaltung der Spielräume auf der Organisationsebene analysiert (vgl. DeMarco 2001; Tröster 2013), so wird heute das Übermaß an persönlicher Freiheit und die mangelnde Fähigkeit des Individuums, sich dieser sinnvoll zu bedienen, als Ursache der sinkenden Produktivität in dem Arbeitsprozess beschrieben. Dieser paradigmatischen Verschiebung liegt die veränderte Wahrnehmung der Organisation von Arbeit, die im Übergang von Taylorismus zum Post-Taylorismus stattgefunden hat, zugrunde.

\subsection{Neue Anforderungen veränderter Arbeitsorganisation}

Das wesentliche Prinzip des Taylorismus bestand in der »Trennung zwischen den Arbeitenden als Subjekt und Arbeitskraft.« (Böhle 2010: 78) Dadurch wurde in den Arbeitsprozessen eine Abgrenzung zwischen Markt und Organisation geschafft: "außerhalb der Arbeit ist man ein selbstverantwortlicher, freier Bürger und in der Arbeit unterliegt man dem Direktionsrecht des Arbeitgebers.«(Ebd.) Das neue Organisationsprinzip des Post-Taylorismus in dem Zeitalter der Technologisierung und Globalisierung richtet sich zum einen darauf, »die Organisation von Unternehmen zu flexibilisieren und zu verschlanken sowie zum anderen darauf, neue Rationalisierungspotenziale im Bereich qualifizierter und verantwortlicher Arbeit zu erschließen.«(a.a.O.: 85) Diese Subjektivierung der Arbeit hat neue Prinzipien in die Steuerung und Kontrolle von Arbeitsprozessen eingeführt: »An die Stelle der direkten Steuerung und Kontrolle der Arbeit - wie dies in der tayloristischen Rationalisierung der Fall war - tritt nun die indirekte Steuerung oder Kontextsteuerung« (a.a.O.: 86), deren Organisationsprinzip sich auf die Vermarktlichung dieser Steuerung bezieht. Durch die paradigmatische Veränderung der Arbeitsprozesse und durch den Fokus auf kontextuelle und indirekte Steuerung, hat sich die Arbeit subjektiviert und ökonomisiert. So sind auch die Bedrohungen und Ursachen für die Belastungen, die aus den Arbeitsprozessen resultieren, nicht sofort erkennbar:

»Die indirekte Steuerung der Arbeitsleistung durch sachliche, zeitliche und personelle Vorgaben sowie Vermarktlichung und Ökonomisierung entziehen sich als Ursache von Belastungen der unmittelbaren Erfahrung. Anders als die Gefährdung der Cesundheit durch Lärm und Hitze oder hohe und einseitige körperliche Anforderungen resultieren die Belastungen bei der Subjektivierung von Arbeit aus dem Zusammenwirken unterschiedlicher Faktoren in komplexen Arbeitssituationen: den Arbeitsanforderungen, den verfügbaren Ressourcen, dem internen Wettbewerb, dem Druck des Marktes usw. «(Böhle 2010: 88) ${ }^{9}$

9 Um diese Zusammenhänge erfassen zu können, wurde in der Wissenschaft »anstelle eines eindimensionalen Ursache- und Wirkungsverhältnisses ein rrelationales Belastungskonzept entwickelt.« (Böhle 2010: $88 \mathrm{f}$ [Herv. i. O.]) 
Die indirekte Steuerung von Arbeitsprozessen prägt auch unsere Vorstellung von der Arbeit selbst. Christoph Bartmann beschreibt in diesem Zusammenhang drei Leitmotive, die für die veränderte Wahrnehmung der Arbeit kennzeichnend sind. Zum einen sind wir stets darauf bedacht, unsere Arbeit und den damit verbundenen Höhepunkt unserer Leistung auf die Zukunft hin zu richten: »Den Gipfel unserer Leistungsfähigkeit oder Performance haben wir stets noch vor uns. " (Bartmann 2012: 219) Zum anderen haben wir das Bedürfnis, auf unsere Arbeit hinweisen $\mathrm{zu}$ müssen, denn »wir arbeiten nicht nur [...], wir zeigen oder weisen nach, während wir arbeiten, >dass` wir arbeiten, und sind schon deshalb niemals selbstvergessen.« (a.a.O.: 223 [Herv. i. O.]) Schließlich werden wir in der Arbeit aufgefordert, "'glatte<, visuell-grafische, problem- und widerspruchsfreie Formen der Präsentation« (a.a.O.: 239 [Herv. i. O.]) zu leisten, um den Anforderungen unserer Träger - im öffentlichen Dienst wie im Unternehmen - zu entsprechen. Dadurch entsteht eine Standardisierung von Arbeitsprozessen und Lebensgewohnheiten. Die omnipräsente und erforderliche Leistung (Performance), Hinweisung (Indikation) und Selbstdarstellung (Präsentation) haben das Office, den gegenwärtigen Lebensraum, in einen Ort verwandelt, in dem wir als Subjekte unsere Unterdrückung erleben und gleichzeitig unsere Befreiung realisieren (vgl. a.a.O.: 242f). Für diese neue Welt »der Präsentationen ohne Präsenz, der Performance ohne Ereignis, der Indikationen ohne Indiziertes, der großen, leeren Schau« (a.a.O.: 244) zahlen die Menschen einen hohen Preis. Gerade Burnout, die »Krankheit des positiven Denkens, der Optimierungen und der Performance« (a.a.O.: 246), soll das Resultat dieser Entwicklungen sein:

»Erst hat uns das positive Denken glauben gemacht, es gäbe für uns kein Limit, nun begegnen wir uns wieder als Performance-Junkies in der Kurklinik und üben uns, wie man jetzt immer häufiger hört, in >Achtsamkeit«. Die schädlichen Folgen positiven Denkens sollen offenbar durch noch mehr positives Denken behoben werden.«(a.a.O.: $265 f$ [Herv. i. O.])

\section{Soziokultureller Blick auf das Burnout-Syndrom}

Die gesellschaftstheoretische Kritik des Burnout-Syndroms und vergleichender psychischer Störungen, wie Boreout-Syndrom, Borderline, ADHS oder Depression, bezieht sich auf verschiedene Entwicklungen des modernen und postmodernen Zeitalters. Die soziologischen und kultursoziologischen Auslegungen fokussieren insbesondere auf die Verhältnisse, in die das Individuum eingebettet ist und die seine Subjektformen bestimmen. 